\title{
Procedural simulation method for aggregating charging load model of private electric vehicle cluster
}

\author{
Mingfei BAN ( $\varangle)$, Jilai YU
}

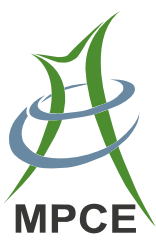

\begin{abstract}
The usage of each private electric vehicle $(\mathrm{PrEV})$ is a repeating behavior process composed by driving, parking, discharging and charging, in which PrEV shows obvious procedural characteristics. To analyze the procedural characteristics, this paper proposes a procedural simulation method. The method aggregates the behavior process regularity of the PrEV cluster to model the cluster's charging load. Firstly, the basic behavior process of each PrEV is constructed by referring the statistical datasets of the traditional private non-electric vehicles. Secondly, all the basic processes are set as a simulation starting point, and they are dynamically reconstructed by several constraints. The simulation continues until the steady state of charge (SOC) distribution and behavior regularity of the PrEV cluster are obtained. Lastly, based on the obtained SOC and behavior regularity information, the PrEV cluster's behavior processes are simulated again to make the aggregating charging load model available. Examples for several scenarios show that the proposed method can improve the reliability of modeling by grasping the PrEV cluster's procedural characteristics.
\end{abstract}

Keywords Electric vehicle (EV), Private electric vehicle (PrEV), Charging load model, State of charge (SOC), Procedural simulation, Cluster

CrossCheck date: 27 February 2015

Received: 30 September 2014/ Accepted: 18 February 2015/ Published online: 7 May 2015

(C) The Author(s) 2015. This article is published with open access at Springerlink.com

M. BAN, J. YU, Harbin Institute of Technology, Harbin 150001, HLJ, China

(凹) e-mail: banmingfei@hit.edu.com

\section{Introduction}

Large scale deployment of electric vehicles (EVs) will provoke considerable impacts on the power system [1]. It is essential in studying the impacts and how to take advantages of EVs to provide ancillary services [2]. And aggregating the charging load model for EVs becomes a crucial problem.

A number of approaches have been proposed to forecast the charging load of EVs. In [3-5], the trip distances, initial stage of change (SOC) and charging time were simulated by several independent probability distributions, and then the charging load model was established. In [6], based on the data provided by GPS devices, more accurate results were obtained by conditional probability distribution function. Besides, to consider the stochastic natures of EV transportation variables, a joint distribution function with copula functions was developed in [7]. In [8], a spatialtemporal model based on intelligent transportation researches was proposed, and the origin and destination (OD) analysis was introduced to model the mobility of EVs. In [9], the diversity of vehicle users' using habits is considered, and its modified model, which considers the effect of road slope, was presented in [10]. The distribution of EVs' parking demand was discussed in [11], and then the spatial and temporal distribution of EVs' charging load was studied. In [12], an EV is assumed to be charged immediately while it parks, and the charging will not stop until it is fully charged or its next trip begins, then the daily charging load curves of various typical EVs were achieved. Potential EV users were screened out by empirical analysis in [13], and their characteristics were defined as the basic indicators to predict the charging demand of future EVs. In [14], an analytical method based on the non-homogeneous semi-Markov processes was proposed to model the charging behavior of EVs. The usefulness of the National 
Travel Survey was investigated in [15], and a generic charging load modeling method, which considers the detailed using habits of EVs, was proposed.

Current researches have been done with lots of active explorations. However, although the methods in [4-7] are easy to implement, their results are not credible enough, as they lack effective methods to model the inherent randomness of EVs. Despite various improvements have been presented in [8-11], the behavior characteristics of EVs are still described by traditional analytical methods. On the other hand, the method in [12] is clear and articulate, but it is too idealistic to consider the complex driving and charging behavior of EVs. As for [13-15], the characteristics of EVs may well be simulated by using the historical data, but it is an arduous task to collect the large amounts of essential data. Meanwhile, the simplification they made would have serious impact on the accuracy of the results, thus the role they could play in a specific distribution network still needs further research.

Oversimplification and data collection difficulty impact seriously on the modeling accuracy. Meanwhile, various random factors and the discrepancy between EVs are too complicated to be directly reflected by the statistical results or mathematic analysis.

Additionally, the time windows discussed in most of the related literature are confined to just no more than one day. However, the realistic charging and discharging cycle of an $\mathrm{EV}$, especially a PrEV, is much longer. Hence it is prone to misalignment in describing the EV cluster's SOC and behavior process, which will result in serious distortions in modeling.

The characteristics of different EVs are distinctive, specifically, we only studies the PrEV in this paper. PrEVs usually have different behavior processes on weekdays and weekends, and the majority of them can complete one charging-discharging process within one week, herein one week is assigned as the time window.

The proposed method could depict the behavior of a PrEV in a long time window. And based on readily accessible data, it can effectively aggregate the behavior process regularity and SOC distribution of the PrEV cluster from individual level. Then by simulating the continuous driving and charging process of each PrEV, the total charging load curves are obtained.

The remainder of this paper is organized as follows: Sect. 2 describes the procedural characteristics of the PrEV cluster and the overall modeling approach. Section 3 covers the information about the reference datasets and related parameters. Sections 4 and 5 study the PrEV cluster's behavior process regularity and SOC distribution respectively. Section 6 introduces the aggregated model and the cases study, and then the conclusions are given in Sect. 7.

\section{Procedural characteristics and modeling approach}

\subsection{Procedural characteristics analysis}

Generally, similar to the non-electric private vehicles, the travel destinations of PrEVs can be classified into three categories [14]: (1) Home, (2) Work, (3) Else. And as shown in Fig. 1, the behavior process of a PrEV can be depicted by several basic driving routes, Route 1 to 4 , or their combinations.

A PrEV's behavior can be portrayed by two typical processes. One is the daily driving and parking process, which cycles day by day. The other is the charging and discharging process, which tends to have a longer cycle. The latter usually contains several ones of the former. As the two processes continuously interweave in the time domain, it provides a good basis for aggregating the charging load model of the PrEV cluster with procedural simulation.

Since historical data can not directly provide detailed discharging-charging data of the PrEVs, it is important to make rational use of the PrEV cluster's procedural behavior characteristics to obtain necessary information.

With the promotion of the charging facilities at workplaces and public parking stations, the charging behaviors of PrEVs will become more flexible and diversified. However, due to the time-of-using price and the customer habits, most charging will take place after the daily last trip [16]. To facilitate subsequent analysis, the following assumptions and simplifications are considered.

1) PrEV's charging only takes place after the daily last trip, and only at home or the residential area.

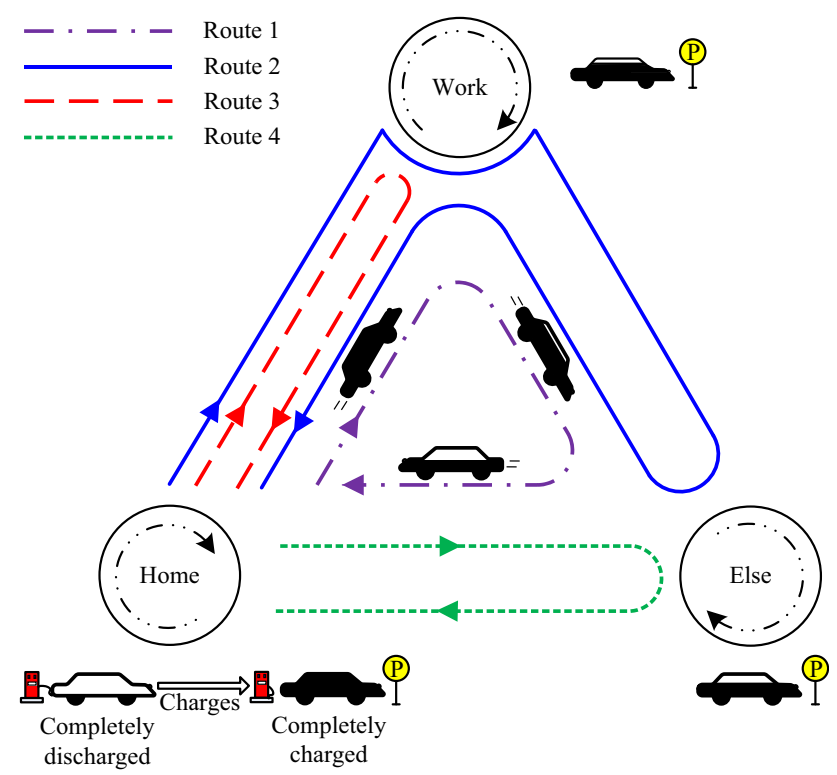

Fig. 1 PrEVs' typical process of mobility 




Fig. 2 Typical behavior processes of a PrEV

2) PrEV's usage is classified into two patterns: weekdays and weekends.

3) Bidirectional energy transferring modes, such as V2G [17] and V2B [18], are not considered.

Then, a PrEV's behavior processes before the daily last trip mainly influence its total trip distance, which can be reflected by its SOC decrease. In practice, we mainly consider the arriving moment of the last trip and the SOC before charging, rather than all the details. Thus the PrEV's behavior processes before the daily last trip can be simplified, and the remaining processes related with the charging load become even more prominent.

Figure 2 shows the typical behavior process of $\mathrm{PrEV}_{i}$ at day $j$. It can be simply portrayed by SOC and a series of serialized time indicators. In Fig. 2, $S O C_{i, j}$ is the $\mathrm{SOC}$ of $\mathrm{PrEV}_{i}$ when it finishes the $j^{\text {th }}$ day last trip, and $S O C_{\mathrm{O} i, j}$ is the original SOC of $\mathrm{PrEV}_{i}$ before the first trip's, while $S O C_{\mathrm{MAX} i}$ is the maximum $\mathrm{SOC}$ of $\mathrm{PrEV}_{i}$. And the serialized time indicators include the departing moment $t_{i, j-\mathrm{b}}$, the midway sojourn time $T_{i, j-\mathrm{s}}$, the driving time $T_{i, j-\mathrm{t}}$, the arriving moment $t_{i, j-\mathrm{a}}$, the delay time $T_{i, j-\mathrm{d}}$ and the charging starting moment $t_{i, j}$.

Since each time indicator could represent a time period or a time period's starting/ending point in the behavior process of a PrEV, the serialized time indicators may well portray a PrEV's behavior process. Hence, grasping these indicators is helpful to grasping the behavior process regularity of a PrEV.

\subsection{Overall modeling approach}

The modeling approach consists of two consecutive parts as shown in Fig. 3. The first part aims at aggregating information about the SOC and serialized time indicators for all the PrEV individuals. And the second part models the charging load of the cluster based on the converged solution of the first part.

1) Part 1: In order to get the SOC and serialized time indicators of the PrEV cluster, the temporary behavior process of each PrEV individual is iteratively structured and simulated. By dynamically calibrating and restructuring the processes in each procedural simulating cycle, the regular distributions of the SOC and serialized time indicators for the PrEV cluster are gradually refined. This part includes the following two parallel sub-processes.

Sub-process 1: To study the behavior process regularity of the PrEV cluster, several data-subsets about the time indicators and trip distance are generated. And the samples are extracted from them to characterize a temporary behavioral process for each PrEV individual. Then procedural simulation is conducted on these temporary behavior processes, and the simulation results are compared with the actual surveys. The untrustworthy ones are dynamically updated and reconstructed to make the total behavior process regularity of the PrEV cluster more consistent with actual statistics. Its detailed process is given in Sect. 4.

Sub-process 2: To study the SOC distribution of the PrEV cluster, driving and charging processes of all the PrEVs are continuously simulated week after week. Then the daily SOC information of each PrEV is available, and the overall SOC distribution of the PrEV cluster is dynamically monitored and saved. The simulation ends when the SOC distribution tends to stabilize within appropriate criterions. More details are described in Sect. 5.

2) Part 2: Taking the SOC distribution and the behavior process regularity information obtained in Part 1 as initial and constraints respectively, a new simulation begins. The driving and charging and process of each PrEV is continuously simulated. Meanwhile, various random influencing factors and different charging scenarios are embedded. In this way, the SOC and charging starting moment information of each PrEV individual becomes available. Then the charging load curves of the PrEV cluster can be obtained.

\section{Datasets and parameters}

\subsection{Datasets}

To get the serialized time indicators and SOC distribution of the PrEV cluster, statistical data about the driving 




Fig. 3 Framework of the overall modeling approach

behavior are extracted from the 2011 Transportation Report provided by the Beijing Transportation Research Center [19]. The datasets include information about the trip distance, departing moment, midway sojourn time, arriving moment, traffic conditions and so on.

It should be pointed out that the data utilized in this paper are easily accessible, and can also be obtained from other practical traffic statistics for specific area, thus the applicability of the proposed method is enhanced.

\subsection{Time indicators related parameters}

As shown in Fig. 2, if $\operatorname{PrEV}_{i}$ charges on the $j^{\text {th }}$ day, its charging starting moment $t_{i, j}$ is decided by the arriving moment $t_{i, j-\mathrm{a}}$ and the delay time $T_{i, j-\mathrm{d}}$, which can be described as

$t_{i, j}=t_{i, j-\mathrm{a}}+T_{i, j-\mathrm{d}}=t_{i, j-\mathrm{b}}+T_{i, j-\mathrm{s}}+T_{i, j-\mathrm{t}}+T_{i, j-\mathrm{d}}$

In (1), $t_{i, j-\mathrm{b}}$ and $T_{i, j-\mathrm{s}}$ derive from traffic statistics [19], and $T_{i, j-\mathrm{s}}$ is determined by sojourn behavior. In general, sojourn behavior can be fixed (i.e. sending and picking up children) or random (i.e. shopping, visiting) [19]. $T_{i, j-\mathrm{d}}$ is determined by the charging scenarios. While, the driving time $T_{i, j-\mathrm{t}}$ is related with the last trip distance $S_{i, j}$ and the driving speed $v_{i, j}$.

For $\operatorname{PrEV}_{i}, S_{i, j}$ can be defined as sum of half the daily basic trip distance $d_{i, j}$ and the random additional trip distance on the way home $d_{+i, j}$. Basic trip distance probability density function on weekdays $y_{\mathrm{wd}}[20]$ and weekends $y_{\mathrm{we}}$ $[19,20]$ can be described by

$$
\left\{\begin{array}{l}
y_{\text {Wd }}=\frac{1}{b^{\mathrm{a}}(a-1) !} x_{1}^{a-1} e^{-\frac{x_{1}}{b}} \\
y_{\mathrm{We}}=\frac{r}{\sigma_{1} \sqrt{2 \pi}} \mathrm{e}^{-\frac{\left(x_{2}-\mu_{1}\right)^{2}}{2 \sigma_{1}^{2}}}+\frac{1-r}{\sigma_{2} \sqrt{2 \pi}} \mathrm{e}^{-\frac{\left(x_{2}-\mu_{2}\right)^{2}}{2 \sigma_{2}^{2}}}
\end{array}\right.
$$

where $x_{1}$ and $x_{2}$ are the trip distance on weekdays and weekends respectively; $a$ the shape parameter; $b$ the scale parameter; $r$ the weight of bimodal-normal distribution; and $\sigma_{1}, \sigma_{2}, \mu_{1}, \mu_{2}$ the standard deviations and expectations of corresponding normal distributions respectively.

$d_{+i, j}$ is related with the random sojourn behavior and can be determined by corresponding distribution. It has little effect on the modeling result, thus we do not place emphasis on it.

In addition, in order to consider the impact of main random factors on the modeling, no trip and long-distance extra trip are considered. The proportions of PrEVs with no trip on weekday and weekend are $r_{\mathrm{nw}}$ and $r_{\text {ne }}$ respectively, and the corresponding trip distances are 0 for the day. The proportions of PrEVs with long-distance extra trip on weekday and weekend are $r_{\mathrm{aw}}$ and $r_{\mathrm{ae}}$ respectively, and their trip distance can be described by normal distribution $N_{1}$ and $N_{2}$.

As for $v_{i, j}$, it can be given by the fitting function of traffic survey. Yet for PrEVs with sojourn behavior, it is hard to accurately describe $v_{i, j}$. To expedite following simulation, the sojourn behavior is assumed to occur in the middle of the last trip.

Then $t_{i, j-\mathrm{a}}$ can be obtained from

$\int_{i, j-\mathrm{b}}^{t_{i, j-\mathrm{a}}} v_{i, j}(t) \mathrm{d} t=S_{i, j}$

\subsection{SOC related parameters}

$S O C_{i, j}$ is determined by $\mathrm{PrEV}_{i}$ 's driving and charging behavior and directly related with the trip distance, which can be described as

$$
\begin{aligned}
S O C_{i, j}= & S O C_{i, 0}-\frac{d_{i, j}}{s_{i}\left(1-\eta_{i}\right)} \\
& +\left(\sum_{h=1}^{j-1} \Delta S O C_{i, h}-\frac{d_{i, h}}{s_{i}\left(1-\eta_{i}\right)}\right)
\end{aligned}
$$

where $S O C_{i, 0}$ is the initial SOC; $s_{i}$ the nominal mileage; $d_{i, h}$ the trip distance at day $h ; \triangle S O C_{i, h}$ the SOC increment at 
day $h$, and $0 \leq \triangle S O C_{i, h} \leq S O C_{\mathrm{MAX} i}-S O C_{i, h}$; and $\eta_{i}$ an efficiency and aging coefficient [21], it is introduced to consider the capacity and mileage decreases of $\operatorname{PrEV}_{i}$, while it can be determined by

$\eta_{i}=f\left(C_{\text {total }}, D_{\text {total }}, T_{\text {total }}\right)$

where $C_{\text {total }}, D_{\text {total }}$ and $T_{\text {total }}$ are the total charging energy, trip distance and usage time respectively, and concrete formula of $f$ could be given based on actual situation.

The criteria for $\operatorname{PrEV}_{i}$ needing to be charged is defined as

$\frac{d_{i, j+1}+l_{i, j+1}}{s_{i}\left(1-\eta_{i}\right)}>S_{O S C} C_{i, j}$

where $d_{i, j+1}$ is the planned trip distance for next day, and it is usually decided by (2) or depends on specific scenario; and $l_{i, j+1}$ the margin, which can be adjusted according to actual cases.

Herein, considering the impact of practical habits of private vehicles owners and battery lifespan, it is assumed that once the charging process starts, it will not abort until the battery is fully charged. For other complex cases, they will be studied further in future work. Then if $\operatorname{PrEV}_{i}$ charges, $S O C_{\mathrm{O} i, j+1}$, the initial $\mathrm{SOC}$ of the next day, equals to $S O C_{\mathrm{MAX} i}$, otherwise it equals to $S O C_{i, j}$.

\section{Behavior process regularity of the PrEV cluster}

For large-scale PrEV cluster, it is hard to directly obtain the behavior regularity of each PrEV. Generally, more realistic approach is to generate several data sub-sets of the serialized time indicators with referring to the traditional non-electric private vehicles and sample corresponding indicator from the data sub-sets for each PrEV to complete its overall behavior process.

However, the approach is not accurate enough as the sampling is random and uncontrolled. Therefore, a procedural simulation method to obtain the basic behavior processes regularity of the PrEV cluster is presented in this section.

\subsection{Modeling approach}

The timeline of one typical day is divided into $m$ sequential segments, and in subsequent work, the processes are conducted segment by segment. Then the presented method is illustrated in Fig. 4, it mainly consists of the following steps.

1) Data preparation: Two data sub-sets about the basic trip distance and departing moment are generated respectively. They have the same size with the PrEV cluster. Then all the departing moment data are classified into the corresponding time segments (from 1 to $m$ ).

2) Constraints setting: When it progresses to the $l^{\text {th }}$ segment, to improve the accuracy and credibility, several constraints are set. These constraints are related with the specific circumstances of the $l^{\text {th }}$ segment, including the average speed, the average trip distance and the ratio of in-transit vehicles, etc.

3) Data combination: For the $l^{\text {th }}$ segment, according to the traffic survey, a certain percentage of the departing moment data are extracted, and the related PrEVs are assumed to have sojourn behavior. Meanwhile, the sojourn time range is given. Thereby, the data combinations of departing moment and midway sojourn time become available. After that, the basic trip distance data are extracted, according to the constraints, and added to these data combinations. Then the roughly driving time can be calculated, and the temporary behavior process sample for each PrEV, whose departing time distributes in the $l^{\text {th }}$ segment, can be assembled.

4) Data inspection: Based on the samples above, the number of PrEVs arrived in the $l^{\text {th }}$ segment is obtained. It is inspected that if its proportion of the PrEV cluster is consistent with statistics. Namely if the error $e_{l}$ is less than the reference $e_{l}^{\prime}$, the serialized time indicator samples are retained and they can represent the basic behavior regularity of the PrEVs distributed in this segment. Otherwise, the samples with their arriving times in the center part of this segment, namely the credible part, are saved to improve the odds of success in the next simulation cycle, and this segment will be reprocessed.

5) Reverse process: When it comes to the last segment, if the remaining data do not meet the accuracy requirements, the samples in previous segments will be added. Then from the last segment to the $1^{\text {st }}$ segment, similar iteration and combination will be conducted. When the requirements of all the segments are met, the temporary process regularity of the PrEV cluster is obtained.

6) Procedural simulation and calibration: Based on the temporary behavior regularity, procedural simulation is conducted. In each simulation cycle, each segment's uncertain factors, such as random sojourn behavior are re-distributed, and the departing moment of each PrEV is set to fluctuate within a certain range. And the untrustworthy ones are dynamically updated and reconstructed to make the total behavior process regularity of the PrEV cluster more consistent with actual statistics. This procedural simulation process 


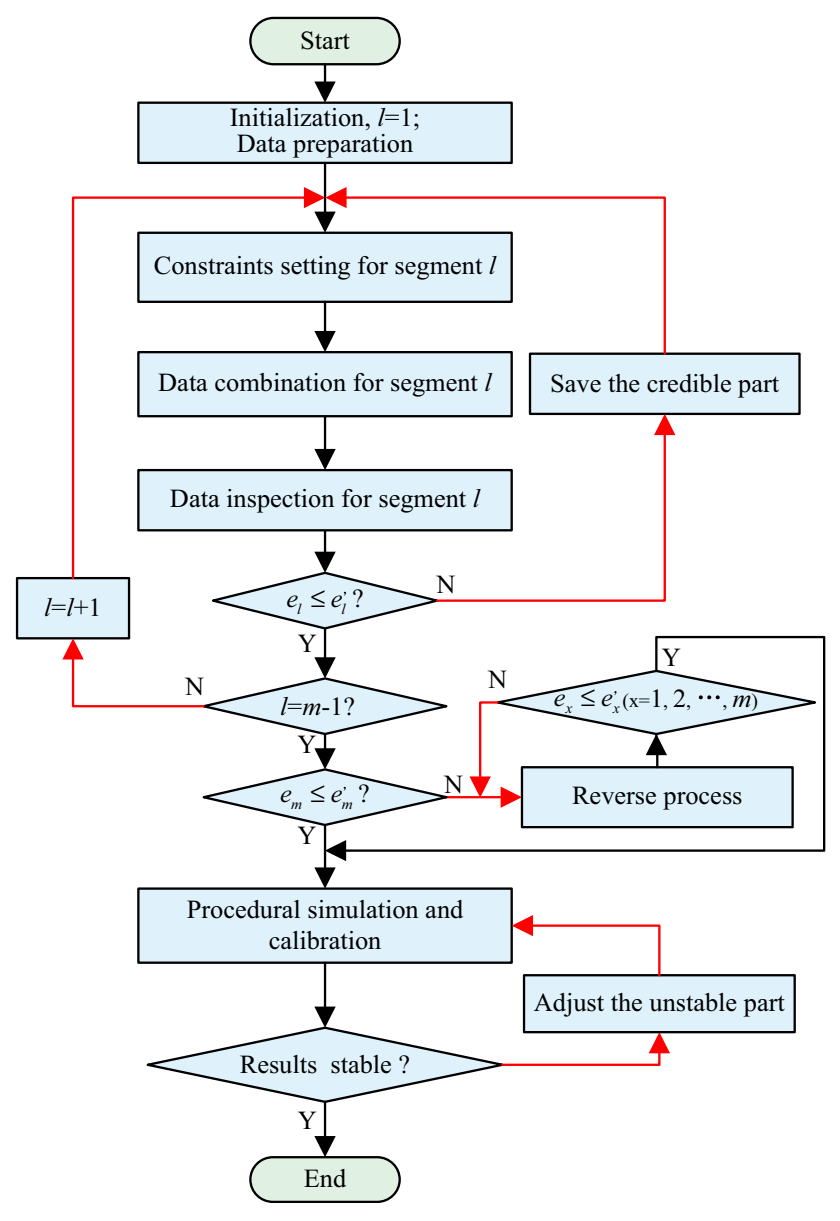

Fig. 4 Flow chart of modeling the behavior process regularity

will be implemented again and again till the last several inspection results are stable.

Finally, the stable serialized time indicators of each PrEV are obtained, and then the PrEV cluster's behavior process regularity can be represented.

\subsection{Simulation cases}

Referring to [19], parameters are set as follows: $a=3$, $b=9 \mathrm{~km}, r=0.65, \sigma_{1}=6 \mathrm{~km}, \mu_{1}=20 \mathrm{~km}, \sigma_{2}=15 \mathrm{~km}$, $\mu_{2}=80 \mathrm{~km}$. When taking a weekday as example, according to the departing moment, the PrEVs are divided into three parts, 16:00-18:00, 18:00-20:00 and 20:00-next day. Assuming that there are 5000 PrEVs in the cluster, and $35 \%$ and $25 \%$ PrEVs in the first two parts have relatively fixed sojourn behavior, and their sojourn time evenly distributed between 10 and $40 \mathrm{~min}$. The simulating result is shown in Fig. 5.

Simulation result shows that the obtained distribution is broadly consistent with actual statistical results. It indicates the behavior process regularity of the PrEV cluster



Fig. 5 Simulation result

obtained from procedural simulation is close to the actual situation.

\section{SOC distribution}

The driving and charging behaviors of PrEVs are relatively regular, thus the overall SOC distribution of PrEV cluster is bound to stable. In this part, a procedural method is presented to reveal the regularity of the SOC distribution.

\subsection{Modeling approach}

The flow chart of modeling the SOC distribution of the PrEV cluster is given in Fig. 6 , in which $[a]$ represents the integer part of $a$, and $i=1,2,3 \ldots, n$.

To start with, a group of rational SOC values are generated to serve as initial for the studied PrEV cluster. To facilitate aggregating a comprehensive model, the vehiclenumbers and corresponding basic trip distances of Sect. 4 are still employed. Besides, parts of the PrEVs are set to have no-trip or long-distance trips in each simulation cycle. Then procedural simulation is conducted day by day to study the SOC information of each PrEV.

During the simulation, the daily SOC distribution information of the PrEV cluster, namely the daily SOC of each PrEV, is saved in every week unit. The SOC distributions in the last $k$ weeks are compared with each other. If the daily SOC distribution differences of the same day among these weeks are all maintained within a certain range, including expectation, variance and proportion of each sub-interval, it is determined that the SOC distributions of the PrEV cluster have tended to stabilize. Otherwise, the above simulation continues. If the daily SOC distributions of the PrEV cluster sustain convergent continuously, it is safe to say the distributions are closer to actual situations. Thus they can be applied to the followup researches to remove the adverse initial effects of defining the SOC distribution with some probability assumptions. 




Fig. 6 Flow chart of modeling the SOC distribution

\subsection{Cases study of the SOC distribution}

Herein, taking Nissan Leaf and BYD E6 (two types of EV) as examples respectively, the parameters are given in Table 1, and the aging affect is ignored.

Considering the characteristics of battery, the SOC is set to range between [0.2-0.9] [22]. Setting $k=5$ and the differences between the expectation and variance of the PrEV cluster's daily SOC distribution less than $5 \%$ as the simulation termination criterion. The other relevant parameters are the same to those used in Sect. 4. For simplicity, PrEVs with random behavior are assumed evenly distributed and randomly selected every day. Besides, $l_{i, j}$ is set to be $d_{i, j+1} / 4$, and ranges between 10 to $20 \mathrm{~km}$.

Figure 7 shows the density distribution of the PrEV proportion about SOC. It can be seen that the results of Nissan Leaf in the 15th and 24th weeks are basically consistent, which verifies the existence of the steady distribution of the PrEV cluster's SOC and the validity of the
Table 1 Parameters of typical EVs

\begin{tabular}{lll}
\hline EV type & Capacity $(\mathrm{kWh})$ & Mileage $(\mathrm{km})$ \\
\hline BYD E6 & 64 & 290 \\
Nissan leaf & 24 & 160 \\
\hline
\end{tabular}
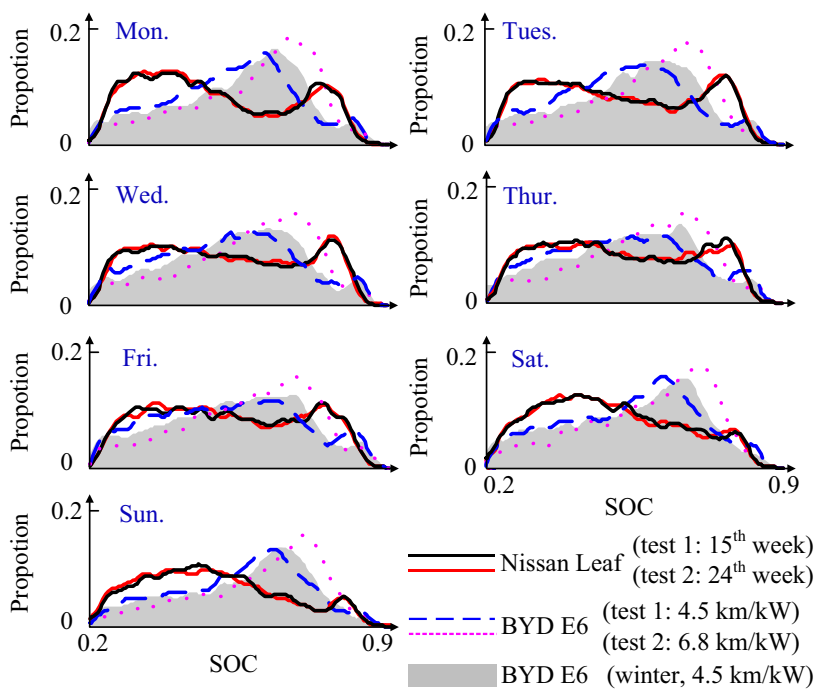

Fig. 7 Simulation results of the SOC distribution

method. In addition, the daily distribution differences of Monday to Sunday are significant, which directly impacts on the modeling of the charging load. However, it is rarely involved in current researches.

Furthermore, there are obvious differences between EVs with different parameters, such as Nissan Leaf and BYD E6. And if the energy efficiency of BYD E6 is improved, for example from 4.5 to $6.8 \mathrm{~km} /(\mathrm{kWh})$, or just assuming that its energy consumption increases by an average of $20 \%$ in winter due to traffic complicacy and heating, its SOC distribution significantly varies. Therefore, the effects of energy conversion efficiency and non-driving energy consumption are revealed, showing that the proposed method can address various factors and has better applicability.

\section{Aggregated model and cases study}

\subsection{Aggregated model}

As shown in Fig. 3, the modeling of the aggregated charging load is implemented as follows.

Setting simulation starting on Monday and taking the SOC data obtained from Sect. 5 as initial, the procedural simulation starts with the basic behavior regularity of each PrEV obtained in Sect. 4. 
The driving and charging processes of each PrEV are continuously simulated. And in all stages of the simulation, various related influencing factors can be added to a particular PrEV or a sub-cluster according to their specific behavior characteristics.

Then the daily SOC and arriving moment information of each PrEV can be obtained. Centralizing the information, and taking $5 \mathrm{~min}$ as step, statistical computations of the PrEVs plug-in and plug-off are dynamically performed. Finally, combined with corresponding charging power and scenarios, the total charging load of the PrEV cluster is obtained.

\subsection{Simulating scenarios}

Referring to existing standards in China, the average charging power of community charging posts, normal charging and quick charging in charging station are set as $7.04,12.16$ and $23.94 \mathrm{~kW}$ respectively. Meanwhile, the time-of-using (TOU) price is set as below, peak time: 10:00-15:00 and 18:00-21:00; shoulder time: 7:00-10:00, 15:00-18:00 and 21:00-23:00; valley time: 23:00-7:00(next day). Then the simulating scenarios are set as follows.

1) Scenario A: Assuming that all PrEVs in target area are equipped with charging posts and they charge once they drive back from daily trips. Besides, the charging process will not stop till they are fully charged.

2) Scenario B: In practice, not all of the PrEVs need charging once they drive back home, and there are a considerable proportion of PrEVs have no charging posts. Furthermore, if V2G [17] and V2B [18] are not considered, to reduce the charging frequency, owners tend to charge their PrEV till they have to.

In scenario B, the charging control methods are grouped into the following three categories: (1) Dumb charging, PrEVs plug in immediately when they need charging; (2) Smart charging considering the TOU price; (3) Smart charging considering both of the TOU price and workers' habit (WH).

Moreover, according to whether they have a fixed parking position, the PrEVs can be divided into three groups: (1) Group A, the PrEVs with fixed parking position and self-charging posts. (2) Group B, the PrEVs without fixed parking position but equipped with battery swapping system. (3) Group C, for which centralized charging in charging station is the only choice. Assuming a PrEV in Group C can finish its charging before 22:00, it charges and leaves, otherwise the charging station will take charge of it for both charging and parking till its owner come to pick it up on the next day. According to [19], the proportions of



Fig. 8 Simulation results of scenario A

Group A, B and C are set as $0.55,0.33$ and 0.12 respectively.

\subsection{Simulation results and discussion}

\subsubsection{Scenario A}

When taking the BYD E6 as example, the charging load curves in scenario A are shown in Fig. 8.

Without considering the TOU price, the simulation results are similar to that in [3-5]: charging load curves on weekdays and weekends are almost identical, which will increase the burden of distribution network, because the charging peak load of the PrEV cluster overlaps with the original one of the distribution network.

When the TOU price is introduced, the charging load peak moves to the starting point of valley price. Even though assuming that all the PrEVs start charging uniformly within half an hour, there is still a sharp load spurt. If all the charging starting points are set just at the same moment when the valley price begins, the load spurt becomes more severe, as shown in the shaded part in Fig. 8. Consequently, it will seriously affect the operation of distribution network.

\subsubsection{Scenario $B$}

The simulation results are shown in Fig. 9. When taking the BYD E6 as an example, the simulation results are shown in Fig. 9a-d is the simulation result for Nissan Leaf.

In Fig. 9a, charging load curves differ from each other, which indicates the necessity to implement a long-term procedural simulation for the PrEV cluster and study its continuous charging load.

In Fig. $9 b$ and $c$, compared with the dumb charging, the smart charging can not only realize a shift of the charging load from the system peak demand time to the valley hours, but also reduce the charging cost. However, the load spurt cannot be ignored. Moreover, the total charging load 


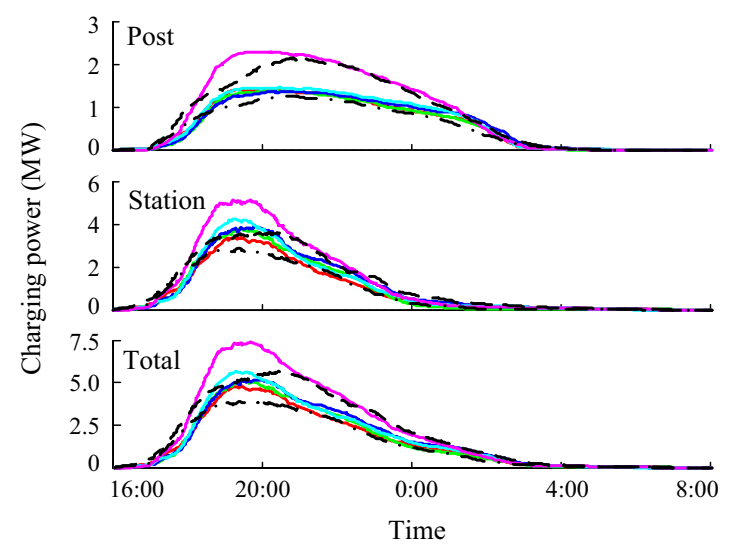

(a) Dumb charging, BYD E6

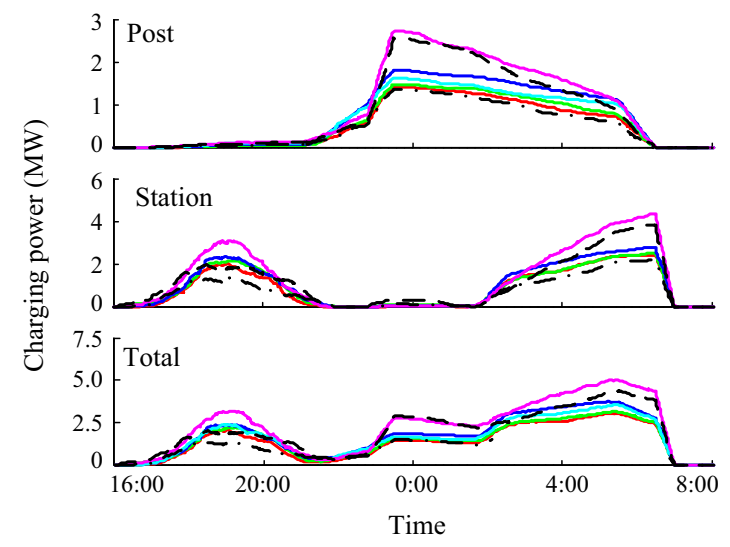

(c) Smart charging with TOU price and WH, BYD E6

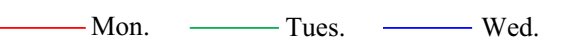

Fig. 9 Simulation results of scenario B

curves in Fig. 9c are smoother than those in Fig. 9b. It follows that the control of the charging load behavior plays a decisive role. With the increasing penetration of PrEVs, it is imperative to research more flexible and feasible charging controlling methods.

Though the charging load curves of the post in Fig. 9b and $c$ are not exactly the same, both of their shapes and trends are broadly consistent. Thus from the perspective of the PrEV cluster, the proposed method can reduce the influence of random factors and increase the reliability of modeling.

Furthermore, for different types of EVs, such as BYD E6 and Nissan Leaf, the simulation results in Fig. 9c and d are distinct. In Fig. 9d, although the differences between loads in a week exist, they are not as significant as that in Fig. 9c. Meanwhile, the total charging demand of Nissan Leaf is obviously less than that of BYD E6. These are consistent with the expectations, as Nissan Leaf has smaller battery capacity but higher energy conversion rate.

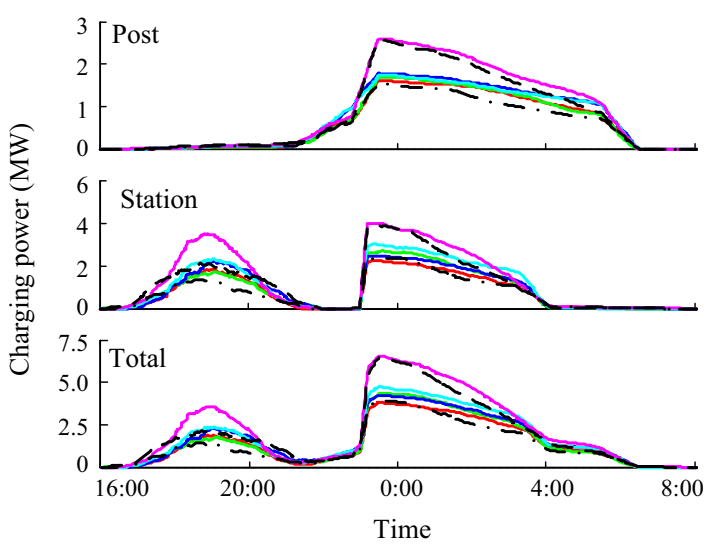

(b) Smart charging with TOU price mode, BYD E6



(d) Smart charging with TOU price and WH, Nissan Leaf Thurs. Fri. - - - - Sat. _...-.. Sun.

\section{Conclusions}

In this paper, the procedural characteristics of PrEV are analyzed and used to aggregate the data about the behavior process regularity and SOC distribution. Then the data are applied in modeling the continuous charging load of the PrEV cluster. The proposed procedural simulation method is simulated in several scenarios, and the results demonstrate its validity.

As each PrEV is independent, the proposed method makes it possible to grasp the behavior process regularity of the PrEV cluster from individual level. And the cumulative effect of the random influencing factors is fully considered in different components of the simulation. Meanwhile, due to its flexibility, it may serve as an analysis tool for the intelligent charging control researches.

The proposed method can effectively track the development of EVs by adjusting the procedural simulation. And it can not only be used in the planning and optimal 
operation for a certain charging station, but also provide reliable reference for prediction and analysis of a specific distribution network.

Future work forecasting.

Acknowledgment This work is jointly supported by the National Natural Science Foundation of China (No. 51377035) and NSFCRCUK_EPSRC (No. 51361130153).

Open Access This article is distributed under the terms of the Creative Commons Attribution 4.0 International License (http:// creativecommons.org/licenses/by/4.0/), which permits unrestricted use, distribution, and reproduction in any medium, provided you give appropriate credit to the original author(s) and the source, provide a link to the Creative Commons license, and indicate if changes were made.

\section{References}

[1] Li R, Wu Q, Oren SS (2014) Distribution locational marginal pricing for optimal electric vehicle charging management. IEEE Trans Power Syst 29(1):203-211

[2] Boulanger AG, Chu AC, Maxx S et al (2011) Vehicle electrification: status and issues. P IEEE 99(6):1116-1138

[3] Jung JS, Cho YJ, Cheng DL et al (2013) Monte Carlo analysis of plug-in hybrid vehicles and distributed energy resource growth with residential energy storage in Michigan. Appl Energ 108:218-235

[4] Qian KJ, Zhou CK, Allan M et al (2011) Modeling of load demand due to EV battery charging in distribution systems. IEEE Trans Power Syst 26(2):802-810

[5] Wu D, Aliprantis DC, Ying L (2012) Load scheduling and dispatch for aggregators of plug-in electric vehicles. IEEE Trans Smart Grid 3(1):368-376

[6] Ashtari A, Bibeau E, Shahidinejad S et al (2012) PEV charging profile prediction and analysis based on vehicle usage data. IEEE Trans Smart Grid 3(1):341-350

[7] Lojowska A, Kurowicka D, Papaefthymiou G et al (2012) Stochastic modeling of power demand due to EVs using copula. IEEE Trans Power Syst 27(4):1960-1968

[8] Mu YF, Wu JZ, Jenkins N et al (2014) A spatial-temporal model for grid impact analysis of plug-in electric vehicles. Appl Energ 114:456-465

[9] Shaaban MF, Atwa YM, El-Saadany EF (2013) PEVs modeling and impacts mitigation in distribution networks. IEEE Trans Power Syst 28(2):1122-1131

[10] Shaaban MF, El-Saadany EF (2014) Accommodating high penetrations of PEVs and renewable DG considering uncertainties in distribution systems. IEEE Trans Power Syst 29(1):259-270

[11] Zhang HC, Hu ZC, Song YH et al (2014) A prediction method for electric vehicle charging load considering spatial and temporal distribution. Automat Electr Power Syst 38(1):13-20 (in Chinese)

[12] Alexander M (2011) Transportation statistics analysis for electric transportation. No 1021848, Electric Power Research Institute (EPRI), Palo Alto

[13] Metz M, Doetsch C (2012) Electric vehicles as flexible loads-A simulation approach using empirical mobility data. Energy 48(1):369-374

[14] Rolink J, Rehtanz C (2013) Large-scale modeling of grid-connected electric vehicles. IEEE Trans Power Deliver 28(2):894-902

[15] Rautiainen A, Repo S, Jarventausta P et al (2012) Statistical charging load modeling of PHEVs in electricity distribution networks using national travel survey data. IEEE Trans Smart Grid 3(4):1650-1659

[16] Zhang MX, Zhuang T (2012) Analysis of the measured charging load of electric vehicle in residential area. Power Demand Side Manage 14(3):44-46 (in Chinese)

[17] Kiviluoma J, Meibom P (2011) Methodology for modelling plug-in electric vehicles in the power system and cost estimates for a system with either smart or dumb electric vehicles. Energy 36(3):1758-1767

[18] Pang C, Dutta P, Kezunovic M (2012) BEVs/PHEVs as dispersed energy storage for $\mathrm{V} 2 \mathrm{~B}$ uses in the smart grid. IEEE Trans Smart Grid 3(1):473-482

[19] Beijing Transportation Research Center (2011) Beijing traffic development annual report. Beijing Transportation Research Center, Beijing (in Chinese)

[20] Yang B, Wang LF, Liao CL (2013) Research on power-charging demand of large-scale electric vehicles and its impacting factors. Trans China Electrotech Soc 28(2):22-27 (in Chinese)

[21] Barre A, Suard F, Gerard M et al (2014) Statistical analysis for understanding and predicting battery degradations in real-life electric vehicle use. J Power Sources 245:846-856

[22] Millner A (2010) Modeling lithium ion battery degradation in electric vehicles. In: Proceedings of the 2010 IEEE conference on innovative technologies for an efficient and reliable electricity supply (CITRES'10), Waltham 27-29 Sept 2010, pp 349-356

Mingfei BAN received the B.S. and M.S. degrees in electrical engineering from Harbin Institute of Technology, Harbin, China, in 2011 and 2013, respectively, where he is currently working toward the Ph.D. degree. His research interests include smart grid and electrical vehicles.

Jilai YU received the B.S. and M.S. degrees in Harbin Institute of Technology, Harbin, China, in 1988 and 1990, respectively, and the $\mathrm{Ph} . \mathrm{D}$. degree in North China Institute of Electric Power, Baoding, China, in 1992. He joined the Department of Electrical Engineering in Harbin Institute of Technology in 1992. From 1994 to 1998, he was an Associate Professor with the Department of Electrical Engineering, where he is currently a Professor and the director of Electric Power Research Institute of HIT. His current research interests include power system analysis and control, optimal dispatch of power system, green power and smart grid. 